\title{
Pharmacist Opportunities to Improve Public Self-Medicating Practices in the UAE
}

\author{
Sanah Hasan ${ }^{1}$, Ghazaleh Farghadani², Salaa Khalid Al Haideri², Mohamed Abdelraouf Fathy ${ }^{2}$ \\ ${ }^{1}$ Department of Clinical Pharmacy, College of Pharmacy, Ajman University of Science and Technology, Ajman, UAE \\ ${ }^{2}$ College of Pharmacy, University of Sharjah, Sharjah, UAE \\ Email: *s.hasan@ajman.ac.ae
}

How to cite this paper: Hasan, S., Farghadani, G., Al Haideri, S.K. and Fathy, M.A. (2016) Pharmacist Opportunities to Improve Public Self-Medicating Practices in the UAE. Pharmacology \& Pharmacy, 7, 459-471. http://dx.doi.org/10.4236/pp.2016.711052

Received: October 11, 2016

Accepted: November 14, 2016

Published: November 17, 2016

Copyright $\odot 2016$ by authors and Scientific Research Publishing Inc. This work is licensed under the Creative Commons Attribution International License (CC BY 4.0).

http://creativecommons.org/licenses/by/4.0/ (c) (i) Open Access

\section{Abstract}

Objective: Self-treatment is an important aspect of self-care and one of the vital issues under debate in health care. Self-medication patterns vary among different populations and are influenced by many factors. The objective was to investigate the nature of self-medication behavior among the general public in the UAE and to explore public attitudes, beliefs, and level of knowledge concerning self-medication. Methods: A purposive sample of individuals involved in self-medication who belong to different age, gender, income, education level and health-seeking behaviors. A qualitative approach through individual face-to-face interviews was utilized to investigate participants' behaviors and attitudes towards self-medication; factors influencing decision to self medicate, sources of information on medications, types of conditions for which self-medication is sought and types of medications used. Participant recruitment continued until theme saturation using content analysis. Findings: Three themes emerged from the data: Reasons for self-medication such as costs associated with visiting a doctor, convenience in visiting a pharmacy and perception of simplicity of the condition; Sources of information: pharmacists as a main source in addition to medication leaflets, family role and previous use, and medicines and medicines' use: medicines' use in chronic disease, use of antibiotics, use of herbals and supplements and medicines' use in children. Conclusion: Findings from this study indicate that self-medication behaviors are common among the population due to several reasons. Inappropriate self-medication practices are evident and may compromise patient care outcomes. Pharmacists play a vital role in intervening to optimize the use of medications and patient education regarding self-care.

\section{Keywords}

Self Medication, Minor Ailments, Community Pharmacy, Qualitative Research, United Arab Emirates 


\section{Introduction}

Self-medication is a usual initial patient response to illness, and in many situations, this may be sufficient in treating many conditions. Self-medication is practiced worldwide [1], and may be more common than the use of prescribed medicines [2]. It is an important aspect of self-care behavior and one of the vital issues under debate in health care. It is defined as the use of drugs to treat self-diagnosed disorders or symptoms, or the intermittent or continued use of a prescribed drug for chronic or recurrent disease or symptoms [3].

Several reasons have led to an emerging demand for the availability of medicines to the public without prescription [4] [5]. The pattern of disease has shifted toward chronic diseases with a consequent shift in management from cure to care. Shortcomings in healthcare systems including inaccessibility to healthcare providers, rising cost of medicines and increased dependence on drug therapy for disease management have led to a natural demand for easier access to these medicines [6] [7].

Although some healthcare providers do not favor self-medication, a valid role for self-medication has been acknowledged by several health organizations such as the World Health Organization (WHO) [1]. A positive effect is recognized for self-medication including patient empowerment and independence in minor ailment management [8], increased convenience of care, and reduction in costs to individual patients and governments [9]. Conversely, if medications are used inappropriately, self-medication can result in wastage of resources, possible emergence of resistant pathogens, and adverse drug reactions [10].

In developed countries, where medication use within the classifications of prescription and non-prescription is strictly enforced, people commonly self-medicate for simple ailments. In developing countries, however, prescription-only medicines are not only used to treat simple ailments, but are commonly used by the general public to treat complex chronic conditions, without health professional supervision. Up to date, little is known about the nature of self-medication behavior among the general public in the UAE; the types of illnesses for which the public seeks self-medication and the types of products commonly self-administered. Hence, it is vital to investigate the nature of self-medication in the UAE and explore public attitudes, beliefs, levels of knowledge and behavior concerning self-medication.

Therefore, this study aims to investigate the nature of self-medication behavior among the general public in the UAE including the types of illnesses for which self-medication is sought, and the types of products commonly used to treat them, and to explore public attitudes, beliefs, and level of knowledge concerning self-medication. This will help shed the light on interventions that could help policy makers and health professionals such as pharmacists assure patients undertake safe and appropriate self-medicating practices and possibly improve the services they provide to their patients.

\section{Methods}

Institutional review board approval was obtained from the Research and Ethics Com- 
mittee before conducting the study.

A qualitative approach through individual face-to-face semi-structured interviews was adopted. An interview guide was prepared and contained questions based on review of the literature to assess frequency of pharmacy visitation, types of conditions for which self-medication is sought, the types of medications and products used, where the medications were obtained; a pharmacy or other outlet stores, whether the use of medications was for acute or chronic care and lastly, participants' attitudes towards selfmedication. Other data collected included: age, gender, origin or nationality and level of education.

A purposive sample was recruited to participate in the interviews. Information about the study and its objectives was disseminated to enrolled students at the College of Pharmacy who helped invite friends, family members and neighbors to participate in the study. Participants with different backgrounds and characteristics related to age, gender, nationality and level of education were recruited. Research assistants then contacted volunteering participants to screen for eligibility. Eligibility criteria included: age-18 years or older and self-report of having visited a pharmacy at least once in the past 3 months. All participants were given an Explanatory Statement and invited to participate, and provided consent prior to participating in the study. Participant recruitment continued until saturation of themes.

Between October 2014 and February 2015, the interviews were conducted by three research assistants trained to conduct qualitative interviews. All interviews were audio-recorded and transcribed verbatim by the research assistants; each interview was transcribed by one transcriber and was double checked by a second one. To ensure trustworthiness, the interviews were conducted at the participants' home and no incentives were provided to them. Notes taken were reviewed by the participants for accuracy during the interviews.

Data were analyzed using content analysis. Each research assistant individually conducted a line-by-line coding of the transcripts; developing primary categories capturing main concepts. A coding scheme was used to code the data which was revised as new data were collected. The research assistants then compared results and discussed any discrepancies between codings. Once agreement was reached on data coding, emerging themes were synthesized.

\section{Results}

Thirty interviews were conducted to reach saturation of themes. Demographic details for participants are shown in Table 1. Three themes emerged from data analysis: Reasons for self-medication, Sources of information and, Medicines and medicines' use:

\subsection{Reasons for Self-Medication}

\subsubsection{Cost}

Many participants prefer to visit a community pharmacy as they cannot afford to visit a doctor, some do not have insurance because of its expense; this encourages them to 
Table 1. Individual interview participant information $(\mathrm{n}=30)$.

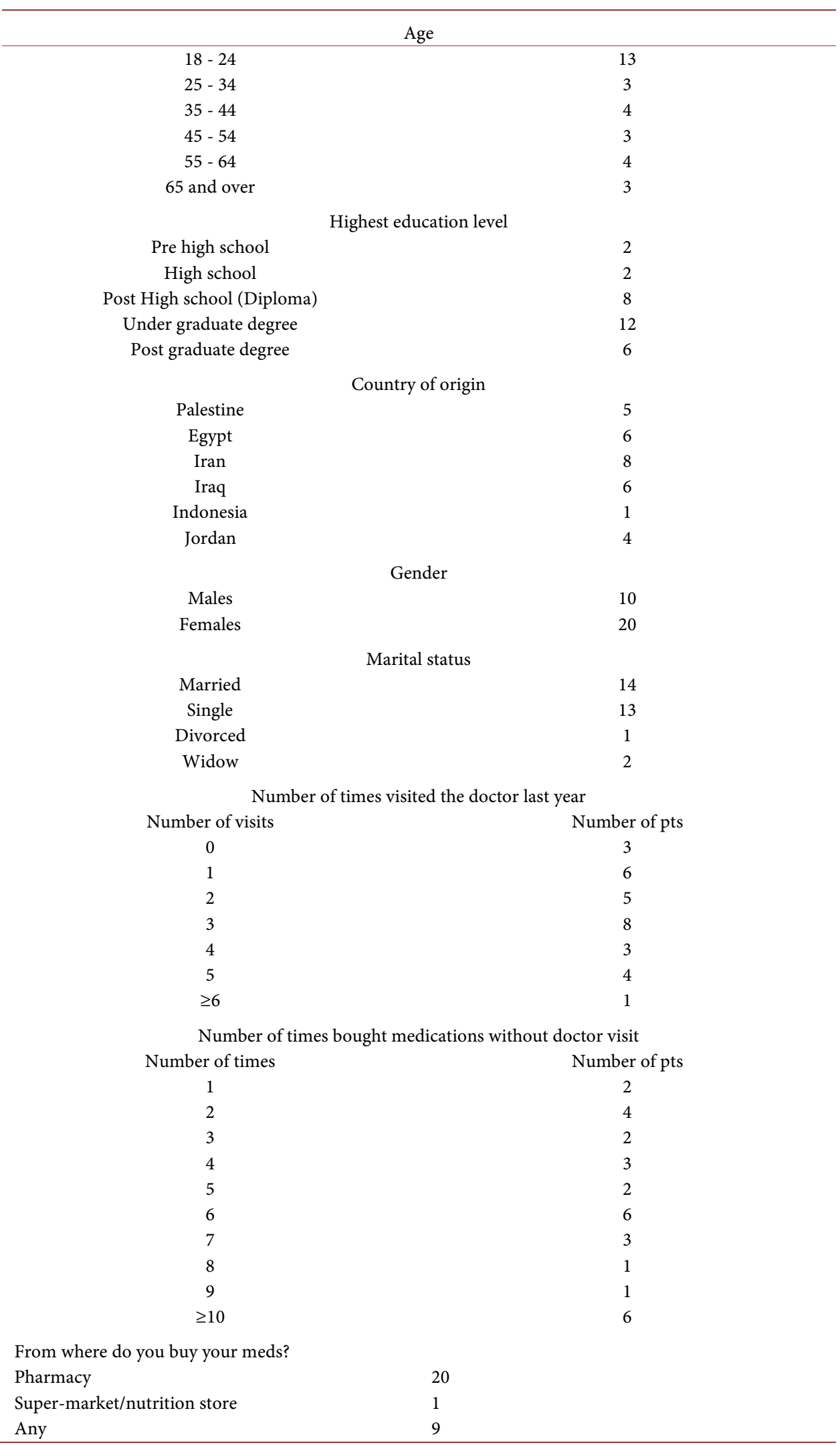


self-medicate:

"Visiting the doctor costs me too much money." SH \# 1

"Self-medication is cheaper... I have no insurance." SA \# 1

\subsubsection{Convenience}

Visiting a community pharmacy offers a convenient way to obtain medications; community pharmacies are available within small geographical distance to most communities, and minimal wait time is required to obtain medications, in comparison to visiting a doctor to obtain a prescription:

"I think it's (visiting a pharmacy) easier and nearer... and I can't find a nearby doctor, and no one to pick me up..." RD\#1

"I don't like going to doctors and waiting." SA\#2

"... it takes time to go to the clinic and wait for the doctor to see him and it really costs a lot if I want to go to doctor for every small issue." SH\#1

\subsubsection{Perception of Simplicity of the Condition}

Many participants reported self-medicating conditions they do not believe to be serious to require medical attention; the conditions being common and easy to self diagnose:

"Normal pain $\cdots$ no need for a doctor." NG\#2

"I always have mild conditions and I think I don't need to go to doctor so I buy the medication only after asking the pharmacist." NR\#1

"It is not a serious condition..." ME\#1

\subsection{Sources of Information}

\subsubsection{Pharmacist's as a Primary Source}

It was apparent that participants more commonly visited community pharmacy than doctors' offices, making the pharmacy a primary source of health and drug-related information:

"In the past year, I visited a doctor maybe once because of skin infection, but I visited the pharmacy more than 10 times in the past six months." NR\#1

"I visit a doctor for the problems that I couldn't manage myself. I visited the pharmacy 6 - 7 times in the last six months!" MR\#1

"I ask the pharmacist... someone (the pharmacist) who studied about something knows better for sure, that's why I ask the pharmacist mostly!” L\#3

Most participants bought their medications from community pharmacies because they trusted the quality of medications there. This also helped patients to access professional services by pharmacists. The majority of participants said they always asked the pharmacist about self treatments because they trust his/her knowledge:

"I only buy my medicines from the pharmacy because I only trust the pharmacy for the quality of the medicines." SOU\#4

"I buy the medicines from the pharmacy only because I want to ask the pharmacist about the medicine." AH\#1 
Despite that most participants said they depend on the pharmacist as their primary source of information about medications, some claimed pharmacists do not give complete information unless it is asked for:

"Sometimes the pharmacists are like sellers, not pharmacists, really, they just give me the med I want without even asking for what I want it... they are not in the mood to talk about the med and explain about it." GH\#1

"They don't even ask what you're taking the medicine for!" GH\#4

\subsubsection{Leaflets and Labels}

In addition to the information pharmacists provide; many participants use the information in the medication leaflet as supplementary to that provided by the pharmacist:

"... sometimes the pharmacist forgets to tell me how to use the medicine, so I ask or use the leaflet." HA\#1

"Sure, I basically read everything in the leaflet!" SOU\#2

"I ask the pharmacist and I read the label and leaflet to have a complete picture!" MEL\#1

\subsubsection{Relationship with Other Health Professionals}

Some participants do not prefer to visit a doctor because of their belief that this leads to routine tests and treatments and those doctors repeat the same tests unnecessarily, possibly complicating the condition:

"They (doctors) keep repeating the same tests and meds that they always prescribe so it's a waste of time and money to go (to the doctor) again." NG\#1

"Doctors do many tests and prescriptions as if they memorized them." HL\#1

"Going to the doctor makes the case more complicated." F\#1

\subsubsection{Previous Use and Familiarity}

Most participants agreed that there was no need to visit a doctor for conditions they encountered previously or for which they had seen a doctor before; they mostly elected to purchase the same medication(s) used earlier:

"... it's from a long time we use these meds and they were always effective in my case." MR\#1

"I take the same treatment as the doctor prescribed me before."

"I had this condition before and I went to the doctor and he gave me the same thing."

\subsubsection{Family Role}

Family plays a big role in the choice of treatments for self-medication. Many participants stated they consulted other family members in diagnosing their conditions and subsequently choosing treatments:

“... mostly from my family, actually my mom's info is very good, she is a phar macist expert." KB\#1 
“... sometimes when we go to the doctor for my little sister and some of the meds are left, I use them when I have the same symptoms." KB\#1

"If I hear from someone that a med is good, I take it. For example my relative said that this pill was good for headache and I went and bought it from the pharmacy." LeHa \#1

\subsection{Medicines and Medicines' Use}

\subsubsection{Safety}

Most participants considered medications for self treatments safe, as they treat simple "clear" ailments:

"It (self-medicating) is safe for simple diseases like cold and flu." NG\#1

Participants reported experiencing adverse effects such as stomachache, diarrhea, dizziness, fatigue and urticaria after using some self-treatments. Some consulted a physician or a pharmacist on the proper management of the side effects, but most either stopped or used another medication:

"The pharmacist changed the medication for me.... and it's ok now, I don't have trouble from this new one..."

"When I got the stomach upset I stopped the medicine..."

"I used another medicine I had at home when I had the itch from augmentin"

\subsubsection{Common Medicines Used}

Participants used a variety of medicines for self-treatment including those to manage chronic conditions and pain, anti-inflammatory and anti-motility agents, antihistamines, decongestants, and a wide range of antimicrobials:

"I buy medicines from pharmacy for my high blood pressure, cholesterol, uric acid, and blood fat." KH\#1

"I normally use: motilium-ceclor-panadol-clarinase-klacid-Benadryl-otrivin: nasal decongestant." NR \# 1

"We have (at home) antibiotics like augmentin, voltaren for pain, brufeen for ear ache, buscopan for stomachache." RD\#1

"The meds I use are usually panadol, panadol cold \& flu, augmentin, naproxen, buscopan, calrinase, fenistel cream, voltaren, histamines for allergy, eye drops for allergy and strepsils." MEL \#1

\subsubsection{Self-Medicating with Antimicrobials}

The majority of participants consume antimicrobials without a doctor's consultation; on previous use, they were found effective by participants. This was commonly facilitated by pharmacists' selling these medications without prescription:

"No I don't visit a doctor, I buy them directly from the pharmacy without visiting the doctor....for throat infections, I ask a pharmacist and he advises me to use them, like augmentin and klacid.” NR\#1 
"I just tell him give me amoxicillin, for example, and they (the pharmacist) never ask for what condition." NG\#1

"When we had Herpes, we went to the pharmacist, he gave us Zovirax." LeHa\#1

"I buy metronidazole and clotrimazole for vaginal problems..." SH\# 1

\subsubsection{Herbs/Supplements}

Most participants used herbal supplements or folk remedies; they believed these products were safe coming from natural sources. Efficacy was not a condition to their use, however; they were considered not harmful if not, for sure, useful:

"Yes I am using iron-multivitamins-ginsana (Ginseng)... they are safe because they are herbs and vitamins." RD\#1

"I use marjoram, sage leaf, ginger, mint and lemon for cough." LJ\#1

"I drink babonnage (chamomile) and ginger with lemon and karkadee (hibiscus) and babonnage (chamomile) with mint..." KH\#1

"I use salvia and chamomile... They are sometimes effective.

"Yes I use them (herbs and supplements) because they are natural $\cdots$ surely, if they don't give benefit, they don't give harm." AU\#1

\subsubsection{Adherence}

Many participants admitted to being non adherent to their treatment recommendations; this ranged from taking more to get a greater and faster effect, to stopping the medicine pre-maturely:

"One time, I took diclofenac $500 \mathrm{mg}$ for bad headache and I slept, after than I felt my tongue is so heavy and I couldn't move it." NG\#1

"Yes, I increase the amount to get a faster effect" ASL\#1

"I stop taking the augmentin, when I feel better." RD\#

\subsubsection{Medicating Children}

Most participants with children treated their young ones for common ailments, including infections. The parents mostly determined the dose based on their prior experience with the medication, while some asked the pharmacist:

"Because they are normal things, we have experience in them from our older kids, so we know these meds, we don't have to see the doctor." SOU\# 1

"For my son, I use nasal drops (decongestants) and emulsions for the flu, flagyl for stomach problems, antibiotics like augmentin, and panadol for cold $\cdots$ I ask the pharmacist if I need to change the amount I need to give.” ABD\# 1

\section{Discussion}

As reported in other studies [11] [12], this study confirmed that costs associated with doctors' office visits, the convenience in accessing community pharmacy and perception of simplicity of the condition were the major reasons for self-medication. In the UAE and many developing countries, access to medical care can be expensive if the in- 
dividual has no medical insurance, which is not uncommon. Additionally, available medical insurance plans may or may not have drug coverage schemes. Cost savings to health services may be a reasonable justification to shift minor ailment consultations to community pharmacy rather than general practice and emergency rooms. Findings from a recent UK report showed that the relative costs for accessing different levels of health care were significantly cheaper through pharmacy services ( $£ 29$ compared with $£ 82$ for general practice and $£ 147$ for emergency hospital visits) [13].

Pharmacists serve as a main source of information for medications used in self treatment. It is obvious that the public trusts pharmacists to supply reliable information on medications. In a previous study of patient expectations of community pharmacy services, participants expressed the need for more information about medications and self care in the UAE [14]. This puts a great deal of responsibility on the shoulders of pharmacists to develop the competency needed to handle patients seeking self treatment. Unfortunately, pharmacists are often accused of being business-oriented which compromises their image as health professionals [15]. Pharmacists should be encouraged to join training programs available locally and internationally that aim to improve competency in caring for self-treating patients.

Participants often used the same medications they previously used for self-treatment; commonly, these medications were previously prescribed by their doctors. Prior prescribed use of medications has been found to lead to self-medicating with leftover previously prescribed medications [16]. Hence, preventing accumulation of leftover medications may be one effective way of reducing inappropriate self-medication. Increasing doctors' awareness that prescribing for minor ailments may increase the risk of subsequent self-medication with the same prescribed medications including antibiotics, and dispensing an exact amount of a drug that corresponds to the amount prescribed are simple but relevant means to reduce inappropriate self medication. This also highlights the need for educating patients about non-adherence which is the most logical explanation for having leftover medications at home. Participants in this study admitted to non-adherence by taking large initial doses to get a faster and stronger effect, and by stopping a medication prematurely as in the case of antimicrobials. Pharmacists need to help patients adhere to their medication schedules and educate them about the consequences of non-adherence including possible adverse events when large doses are consumed and when failing to complete a full course of treatment.

Family role in self-medicating practices has been acknowledged as an important element. Mothers' recommendations have influenced both children and adult self-medication choices [17] [18]. Attitudes, knowledge and behaviors of the mothers themselves concerning self-medication have been shown to influence the nature and outcome of family members' self-medication [19]. Several attempts have been directed towards mothers' education and training in management of medicines available at home for self-medication. One that has been recognized is from Indonesia where mothers and other family members were encouraged to bring in their home medications to a 2 - 3 hour workshop conducted by pharmacists and physicians with the aim of educating family members over common illnesses and the proper use of medicines available at the 
home [19].

Participants admitted to self medicating with a variety of medications ranging from medications for chronic management of hypertension, hyperlipidemia, hyperuricemia, to antibiotics and other inti-infectives, decongestants and antihistamines. In the UAE, there are currently three classifications of medications: prescription only, over-the-counter, and the new pharmacist-only medications [20]. Going with international trends of granting OTC status for some prescription medications, this new classification was designed to facilitate patient access to a variety of medications and give pharmacists legal control over their dispensing. Despite this, prescription-only medications continue to be accessible to the public without prescription through community pharmacy. Regulatory enforcement of the laws prohibiting selling prescription medications without prescription is warranted to assure proper professional follow-up and monitoring. Other medications such as decongestants, antihistamines, nonsteroidal anti-inflammatory drugs, are frequently consumed by the public [21]. It is vital that these medications are not sold indiscriminately to all as a result of their over-the-counter status, or under pressure from patients, as their use could impact chronic disease control.

Several antimicrobials have been reported by most participants as medications consumed commonly for self treatment. Amoxicillin, cefaclor, metronidazole, clarithromycin, acyclovir, clotrimazole are examples of how infections are being treated without proper diagnosis and follow up. Sale of antimicrobials without prescription is common practice in developing countries and contributes to treatment failures and antimicrobial resistance [22] [23]. Pharmacists are in an optimum position to optimize antimicrobial use by preventing indiscriminate access and availability of these medications to the public and by counseling patients on their appropriate use. Adequate enforcement of the laws prohibiting non-prescription sale of antibiotics is due and will help join international efforts currently undertaken to prevent antimicrobial resistance.

Most participants mentioned they used some form of an herbal supplement, multivitamin or folk remedy on a regular basis. Unfortunately, much of the claims these herbal products are marketed for, have not been substantiated making the evidence for their use limited. People using these products often believe that they are, at least, safe if not effective. Evolving literature suggests that not only efficacy claims for many of these products are not justified but also toxicity issues are of major concern [24]. The products used by many of the participants in this study have been reported to cause some side effects; hibiscus has been associated with decreasing both blood pressure and blood sugar [25], chamomile has been associated with allergic-type reactions and drug interactions with warfarin and cyclosporine [26], marjoram (Origanum majorana) can also cause allergies and interacts with diuretics and lithium [27], salvia is generally considered a hallucinogen causing visual perceptions, mood and body sensations, emotional swings and feelings of detachment [28], and ginseng has been associated with agitation, headache, nervousness, trouble sleeping, diarrhea and allergic reactions, and is associated with multiple drug interactions [29]. As patients may not initiate a discussion of herbal products, pharmacists have the responsibility to ask about herbal use and check for possible interactions with patients' use of other herbs or traditional 
medications. This highlights the importance of offering training for pharmacists in herbal product safe use so they could provide comprehensive pharmaceutical care for their patients.

Medicating children by their parents is common all over the world. In a study focusing on antibiotic treatment of children in China, $31.8 \%$ and $34.5 \%$ of the children with common cold and diarrhea were respectively given antibiotics by their parents, again a practice that is inappropriate and promotes bacterial resistance [17]. In another study, it was noted that maternal self-medication with OTC analgesics is associated with self-medication of OTC analgesics, mainly paracetamol among school-aged children, possibly to a larger extent than the child's reported pain [30]. Again, this highlights the need to enforce regulations concerning dispensing prescription-only medications, and the involvement of care givers and family members in discussions about medications requested for self treatment.

This study was the first to report on self medication behaviors in the UAE and to explore public attitudes, beliefs, and level of knowledge concerning self-medication. The findings from this study will help policy makers and health professionals undertake possible interventions and solutions that could assure patients carry out safe and appropriate self-medicating practices. The qualitative approach undertaken in this study may limit the generalizability of the findings due its small sample size. To minimize bias and enhance validity of the data, the interviews were conducted at the participants' homes where privacy and confidentiality were maintained. The participants were aware that the research assistants were working at a college of pharmacy but this has not affected the openness of the discussion.

\section{Conclusion}

The research provided insight about a pivotal issue which needs to be addressed by physicians, pharmacists and UAE authorities. Findings from our study indicate that self-medication behaviors are common among the population due to a variety of reasons and needs. These behaviors are risky at times and may compromise the health of individuals, demanding measures for rational use of medicine. The role of pharmacists in primary care provision and patient education regarding self-care and in developing referral strategies for patients who should not self-treat is highlighted.

\section{Acknowledgements}

The authors would like to thank all participants in this study for their time and openness in expressing frank opinions and thoughts.

\section{Conflicts of Interest}

The authors declare no conflicts of interest.

\section{References}

[1] World Health Organization (WHO) (2000) The Benefits and Risks of Self-Medication: 
General Policy Issues. WHO Drug Information, 14, 1-2.

[2] Cindy, L.K., Maxwell, H.W. and Clarke, M.A. (1989) Survey on the Use of Self-Medication over a Period of Two Weeks. Hong Kong Journal, 11, 371-375.

[3] World Health Organization (2000) Guidelines for the Regulatory Assessment of Medicinal Products for Use in Self-Medication. WHO/EDM/QSM/00.1.

[4] Kitaw, Y. (1997) Self-Care: A Study of Three Communities in Ethiopia. The Ethiopian Journal of Health Development, $2,2$.

[5] Bergmann, J.-F. (2003) Self-Medication: From European Regulatory Directives to Therapeutic Strategy. Fundamental \& Clinical Pharmacology, 17, 275-280.

http://dx.doi.org/10.1046/j.1472-8206.2003.00141.x

[6] Abdelmoneim, A., Idris, E., Lloyd, M. and Lukman, T. (2005) Self Medication with Antibiotics and Antimalarials in the Community of Khartoum State, Sudan. The Journal of Pharmacy and Pharmaceutical Sciences, 8, 326-331.

[7] Clavijo, H.A., Baquero, J.A., Ulloa, S. and Morales, A. (1995) Self Medication during Pregnancy. World Health Forum, 16, 403-404.

[8] Bird, C. and Hassall, J. (1993) Self Administration of Drugs-A Guide to Implementation. Scutari Press, London.

[9] Bond, C. and Hannaford, P. (2003) Issues Related to Monitoring the Safety of Over-theCounter (OTC) Medicines. Drug Safety, 26, 1065-1074. http://dx.doi.org/10.2165/00002018-200326150-00001

[10] World Health Organization (2001) Global Strategy for Containment of Antimicrobial Resistance: World Health Organization, Communicable Disease Surveillance and Response (CSR). WHO/CDS/CSR/DRS/2001.2.

[11] Andualem, T. and Mariam, T. (2004) Self-Medication Practices in Addis Ababa. Ethiopian Journal of Health Sciences, 14, 92-97.

[12] Yousef, A.M., Al-Bakri, A., Bustanji, Y. and Wazaify, M. (2008) Self-Medication Patterns in Amman, Jordan. Pharmacy World \& Science, 30, 24-30. http://dx.doi.org/10.1007/s11096-007-9135-x

[13] Pharmacy Research UK (2013) Community Pharmacy Management of Minor Illness: MINA Study. Final Report, London.

http://www.pharmacyresearchuk.org/waterway/wp-content/uploads/2014/01/MINA-StudyFinal-Report.pdf

[14] Hasan, S., Suleiman, H., Stewart, K., Chapman, C.B. and Kong, D.C.M. (2015) Patient Expectations of and Willingness to Use Primary Care Pharmacy Services in the United Arab Emirates. International Journal of Pharmacy Practice, 23, 340-348.

http://dx.doi.org/10.1111/ijpp.12176

[15] Hasan, S., Stewart, K., Chapman, C.B., Hasan, M.Y. and Kong, D.C.M. (2015) Physicians' Attitudes towards Provision of Primary Care Services in Community Pharmacy in the United Arab Emirates. International Journal of Pharmacy Practice, 23, 274-282. http://dx.doi.org/10.1111/ijpp.12157

[16] Grigoryan, L., Burgerhof, J., Haaijer-Ruskamp, F., Degener, J., Deschepper, R., Monnet, D., et al. (2007) Is Self-Medication with Antibiotics in Europe Driven by Prescribed Use? Journal of Antimicrobial Chemotherapy, 59, 152-156. http://dx.doi.org/10.1093/jac/dkl457

[17] Bi, P., Tong, S.H. and Parton, K. (2000) Family Self-Medication and Antibiotics Abuse for Children and Juveniles in a Chinese City. Social Science \& Medicine, 50, 1445-1450. http://dx.doi.org/10.1016/S0277-9536(99)00304-4

[18] Le, T.H., Ottosson, E., Nguyen, T.K., Kim, B.G. and Allebeck, P. (2011) Drug Use and Self- 
Medication among Children with Respiratory Illness or Diarrhea in a Rural District in Vietnam: A Qualitative Study. Journal of Multidisciplinary Health, 4, 329-336.

[19] Suryawati, S. (2003) Essential Drugs Monitor No. 032. Improving the Quality of Self-Medication through Mothers' Active Learning, CBIA.

http://apps.who.int/medicinedocs/en/d/Js4940e/15.html

[20] United Arab Emirates Ministry of Health. Registration and Drug Control Dept. (2011) Classification-Change of Dispensing Mode.

http://www.cpd-pharma.ae/phocadownload/MOH-Policies-Circulars/MoH-RDCD-Policy\%20Re-Classification-\&-Change-of-Dispensing-Mode-for\%20957-Products-30-Oct-2011-L ist\%201.pdf

[21] Neafsey, P. and Shellman, J. (2000) Adverse Self-Medication Practices of Older Adults with Hypertension Attending Blood Pressure Clinics: Adverse Self-medication Practices. Internet Journal of Advanced Nursing Practice, 5, 1.

[22] Al-Bakri, A.G., Bustanji, Y. and Yousef, Al-M. (2005) Community Consumption of Antibacterial Drugs within the Jordanian Population: Sources, Patterns and Appropriateness. International Journal of Antimicrobial Agents, 26, 389-395. http://dx.doi.org/10.1016/j.ijantimicag.2005.07.014

[23] Li, L.J. and Wang, P.-S. (2005) Self-Medication with Antibiotics: A Possible Cause of Bacterial Resistance. Medical Hypotheses, 65, 1000-1001. http://dx.doi.org/10.1016/j.mehy.2005.05.018

[24] Timbo, B.B., Ross, M.P., McCarthy, P.V. and Lin, C.T. (2006) Dietary Supplements in a National Survey: Prevalence of Use and Reports of Adverse Events. Journal of the Academy of Nutrition and Dietetics, 106, 1966-1974. http://dx.doi.org/10.1016/j.jada.2006.09.002

[25] Drugs.com (2016) Hibiscus. https://www.drugs.com/search.php?searchterm=hibiscus

[26] Drugs.com (2007) Chamomile. http://www.drugs.com/npc/chamomile.html

[27] Vitamins estore.com (2013) Marjoram Leaf Benefits, Reviews and Side Effects. http://www.vitaminsestore.com/marjoram-leaf-benefits-reviews-side-effects-and-dosage/

[28] National Institute on Drug Abuse (2016) Drug Facts: Hallucinogens. http://www.drugabuse.gov/publications/drugfacts/salvia

[29] Drugs.com (2016) Ginseng. http://www.drugs.com/sfx/ginseng-side-effects.html

[30] Jensen, J.F., Gottschau, M., Siersma, V.D., Graungaard, A.H., Holstein, B.E. and Knudsen, L.E. (2014) Association of Maternal Self-Medication and Over-the-Counter Analgesics for Children. Pediatrics, 133, e291-e298. http://dx.doi.org/10.1542/peds.2013-1107 
Submit or recommend next manuscript to SCIRP and we will provide best service for you:

Accepting pre-submission inquiries through Email, Facebook, LinkedIn, Twitter, etc. A wide selection of journals (inclusive of 9 subjects, more than 200 journals)

Providing 24-hour high-quality service

User-friendly online submission system

Fair and swift peer-review system

Efficient typesetting and proofreading procedure

Display of the result of downloads and visits, as well as the number of cited articles

Maximum dissemination of your research work

Submit your manuscript at: http://papersubmission.scirp.org/

Or contact pp@scirp.org 